\title{
Use of pulsed irrigation evacuation in the management of the neuropathic bowel
}

Terry A Puet, Holly Jackson and Sandy Amy

Hillside Rehabilitation Hospital, 8747 Squires Lane, NE Warren, Ohio 44484, USA

\begin{abstract}
Management of the neuropathic bowel is one of the major issues in the treatment of patients with severe spinal cord injury (SCI). Pulsed irrigation evacuation (PIE) has been evaluated in several small studies for the clearing of fecal impactions in patients with a neuropathic bowel. We evaluated our experience with 398 PIE procedures performed on inpatients and outpatients at our facility. It has proven to be both safe and effective in a wide variety of patients with this disorder, and is a useful addition to traditional methods in the management of the neuropathic bowel.
\end{abstract}

Keywords: fecal impaction; neuropathic bowel; spinal cord injury; pulsed irrigation evacuation

\section{Introduction}

Neuropathic bowel is a common and often seriously debilitating problem. However, it is difficult to get a clear idea on the incidence. There is not even a clear definition. The incidence of incontinence has been estimated at 10.9 men and 13.3 women per 100000 population. ${ }^{1}$ The most common cause of the neuropathic bowel is severe spinal cord injury (SCI). There are approximately 250000 persons with SCI and an annual incidence of 10000 new cases yearly in the United States. ${ }^{2}$ A recent review by Krogh found that $80 \%$ of patients with SCI had episodes of fecal incontinence. More importantly $30 \%$ of patients surveyed felt their neuropathic bowel was a more significant problem to them than was bladder dysfunction or sexual dysfunction. ${ }^{3}$

Despite the significance of this problem, there has been much less research and development in the area of bowel management compared to other problem areas in SCI. The evaluation and management of the neuropathic bowel has not changed much in the past 20 years. We previously reported our initial experience with Pulsed Irrigation Evacuation in managing impactions in SCI patients with the neuropathic bowel. ${ }^{4}$ We have recently evaluated an updated device using the same basic technique. The new device, called the PIE 2000, is much smaller, lighter, more portable and significantly less expensive.

\section{Material}

A prototype model of the PIE 2000 was used for this study. It consists of a control unit which measures $4 \mathrm{~cm} \times 5 \mathrm{~cm} \times 2 \mathrm{~cm}$ and is used to control inflow and

Correspondence: TA Puet outflow time. There is also a pack of disposable supplies which includes a water reservoir bag, a cuffed speculum and an outflow bag into which the water and stool flows. This is a closed system designed to minimize fecal soiling and potential contamination. Figures $1 \mathrm{a}, \mathrm{b}$ and $\mathrm{c}$ illustrate the PIE 2000 device.

The general principle behind the PIE is the use of intermittent, rapid pulses of warm water to break up stool impactions and stimulate peristalsis. Enemas have not proven effective in patients with a neuropathic bowel because they cannot usually retain the enema due to decreased sensation and rectal sphincter dysfunction. The use of enemas with cuffed rectal tubes or colostomy irrigation tubes decreases leakage, but there is no control of overdistension of the colon when large amounts of liquid are given before any outflow. The PIE uses a cuffed tube made of silicon to avoid the risk of latex allergy. Inflow is varied from $1 \mathrm{~s}$ to $60 \mathrm{~s}$ which corresponds to $5 \mathrm{cc}$ to $300 \mathrm{cc}$ of $\mathrm{H}_{2} \mathrm{O}$. Outflow time is automatically varied to allow adequate outflow. The reservoir is filled with warm tap water. The speculum is lubricated and inserted. Xylocaine gel is used in patients at risk of autonomic dysreflexia. The procedure is considered complete when the water returns clear, with no further visible fecal material, and generally takes less than $1 \mathrm{~h}$.

\section{Methods}

All patients involved in this study were inpatients or outpatients at Hillside Rehabilitation Hospital. Hillside is a 92-bed freestanding rehabilitation hospital including programs for spinal cord injury, stroke, traumatic brain injury and orthopedic rehab. This paper will focus on spinal cord injury patients, but 
a

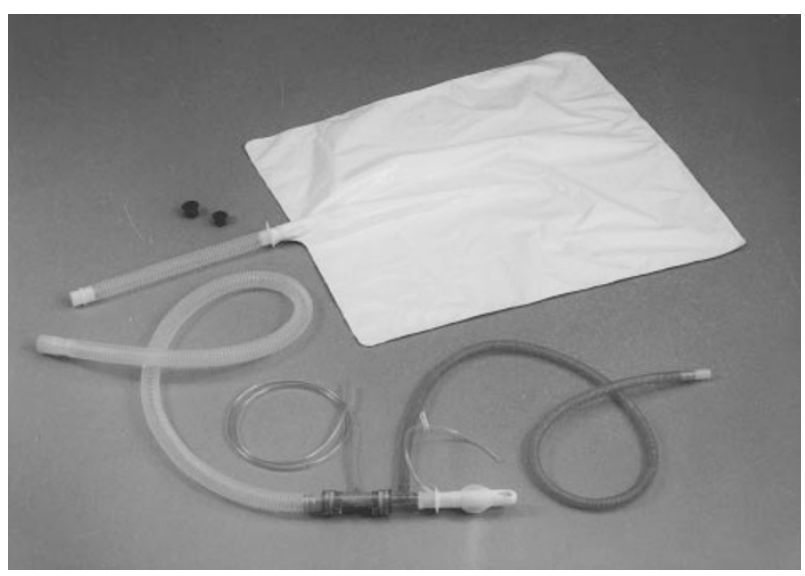

b

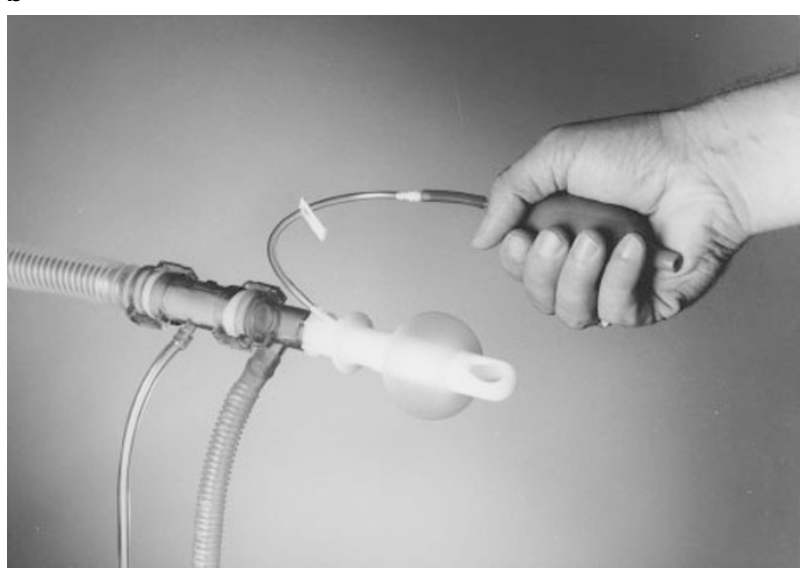

Figure 1

we will also mention the use in other patient populations.

All patients with a spinal cord injury are on a specific bowel routine as ordered by the attending physician. Performance of the bowel routine, charting and monitoring, and patient education are done by the nursing staff. In addition, there is a designated 'bowel and bladder' nurse who assists in managing problem bowel and bladder routines and performs the PIE procedures.

The approach to the standard bowel routine is:

1 find appropriate time for bowel routine to take advantage of gastro-colic reflex;

2 attempt to improve transfers and sitting to allow toileting on commode;

3 give adequate softener;

4 give oral stimulants as appropriate if bowel routine is slow or ineffective;

5 every other day digital stimulation, therevac plus mini-enema, or dulcolax suppository. This may be increased to daily if needed. c

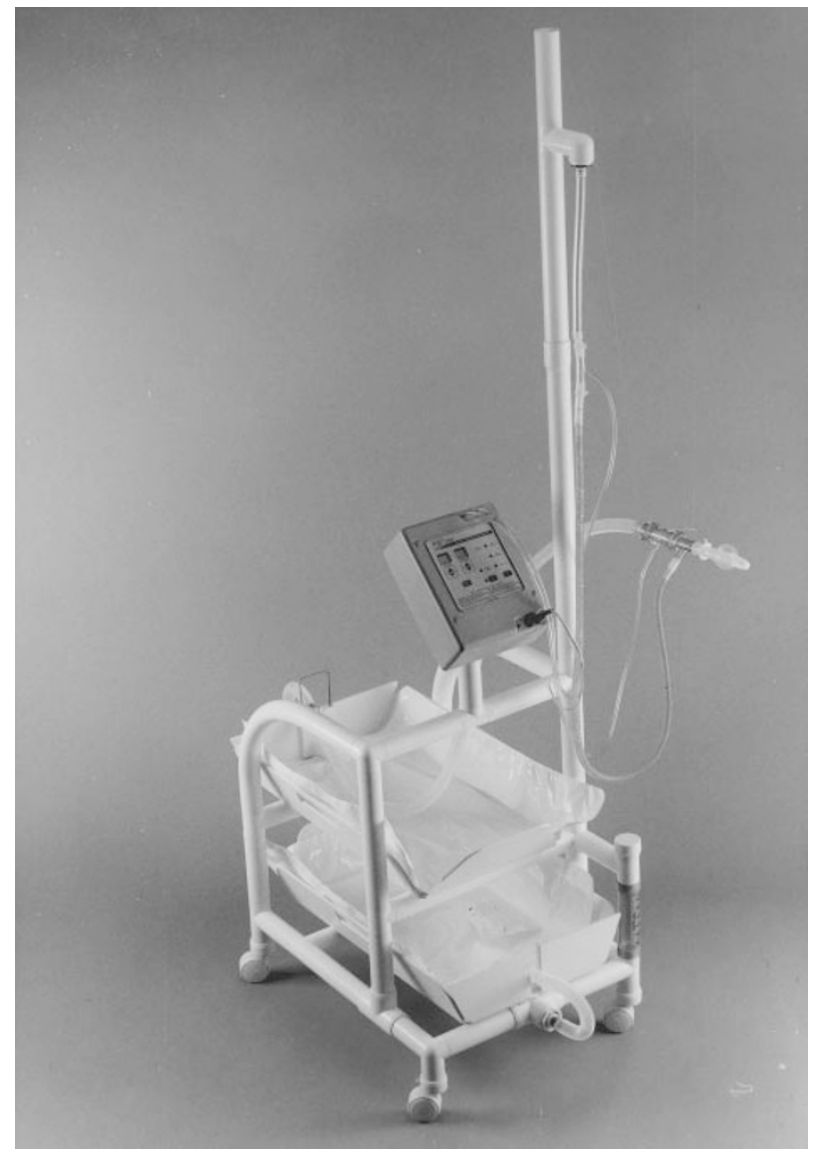

The flow chart summarizes how the PIE is used. It is used basically in three situations:

1 symptomatic impactions with abdominal distension, pain, nausea and vomiting, recurrent liquid stools, autonomic dysreflexia and no response to previous bowel routine;

2 asymptomatic impaction with abdominal distension and no response to bowel routine;

3 failure of the bowel routine to produce stool on three consecutive occasions.

Contraindications: Absolute contraindications we use are:

1 colon surgery within the past year;

2 evidence of acute abdomen;

3 evidence of acute diverticulitis.

Relative contraindications are:

1 history of colon surgery longer than one year ago;

2 history of rectal or lower GI bleeding;

3 history of previous diverticular disease. 
In these circumstances the risk of the procedure was compared to the potential risk of complications from the impaction.

\section{Results}

A total of 398 PIE procedures were done at our facility betwen $1 / 1 / 94$ and $6 / 30 / 96$. Of these, 246 were done on outpatients and 152 were done on inpatients. By diagnoses, 162 or $66 \%$ of outpatient procedures were done on four outpatient SCI patients. These will be looked at in more detail later. Of the 152 inpatient procedures, 63 procedures were done on 31 SCI patients, for a total of $41 \%$. Also $40 \%$ were done on stroke patients and $19 \%$ miscellaneous.

Looking at the 63 procedures done in the SCI population, 20 patients required only one procedure and 11 required procedures on multiple occasions. Two of these patients required four procedures while an inpatient and eventually went on to be long term users of the PIE due to ineffective bowel routines.

Of the 63 procedures, $29(46 \%)$ were done for an ineffective bowel routine, $22(35 \%)$ were done for symptomatic impactions, $12(19 \%)$ were done for asymptomatic impactions (Tables 1, 2 and 3).

Table 1

\begin{tabular}{lcccc}
\hline & $\begin{array}{c}\text { Total } \\
\text { number }\end{array}$ & $\begin{array}{c}\text { PIE } \\
\text { patients }\end{array}$ & $\begin{array}{c}\text { \% requiring } \text { PIE } \\
\text { retal }\end{array}$ & $\begin{array}{c}\% \text { of } \\
\text { SCI PIE's }\end{array}$ \\
\hline $\begin{array}{l}\text { Complete } \\
\text { tetraplegia }\end{array}$ & 15 & 4 & 26 & 13 \\
$\begin{array}{l}\text { Incomplete } \\
\text { tetraplegia }\end{array}$ & 28 & 4 & 14 & 13 \\
$\begin{array}{l}\text { Complete } \\
\text { Paraplegia }\end{array}$ & 35 & 9 & 26 & 29 \\
$\begin{array}{l}\text { Incomplete } \\
\text { Paraplegia }\end{array}$ & 95 & 14 & 15 & 45 \\
\hline
\end{tabular}

Degree of severity of the SCI and the use of PIE

Table 2

\begin{tabular}{rcccc}
\hline & $\begin{array}{c}\text { Total } \\
\text { patients }\end{array}$ & $\begin{array}{c}\text { PIE } \\
\text { patients }\end{array}$ & $\begin{array}{c}\% \text { of total } \\
\text { requiring }\end{array}$ & $\begin{array}{c}\% \text { of } \\
\text { SCI PIE }\end{array}$ \\
\hline $20-40$ & 34 & 11 & 32 & 35 \\
$40-60$ & 23 & 4 & 17 & 13 \\
$>60$ & 116 & 16 & 14 & 52 \\
\hline
\end{tabular}

Age of the patients and the use of PIE

Table 3

\begin{tabular}{lrc}
\hline Total SCI & 173 & \\
Number of patients with PIE & 31 & $(17.9 \%)$ \\
Number of patients PIE $>1$ & 11 & $(6.4 \%)$ \\
Number of patients regular PIE & 2 & $(1.2 \%)$ \\
\hline
\end{tabular}

Frequency of use of PIE
Level of injury

Reviewing patients by diagnoses, we treated a total of 173 patients at our facility with SCI. This number includes traumatic, vascular, tumor, and fracture related spinal cord injuries. It also included patients with cauda equina injuries. Reviewing Table 1, incomplete paraplegic and cauda equina injuries accounted for the largest category of patients, and the largest number of PIE procedures. However, a larger percentage of patients with complete paraplegia and tetraplegia required PIE procedures compared to those with incomplete neural lesions.

Age

Table 2 reviews diagnoses by age. The largest group of patients requiring PIE procedures were those $>60$ years old. However, the largest percentage group requiring the procedure was in the 20-40 year olds. Most of these patients were the traumatic complete cord injuries. In most of the $>60$ year old patients the SCI were related to falls, fractures, tumors or vascular compromise and were more likely to be incomplete.

Safety

To evaluate safety, all of the 398 procedures were reviewed. Only four complications occurred. Two patients became agitated and the procedure had to be discontinued. Both were stroke patients with confusion. Two patients had rectal bleeding on insertion of the speculum. The procedure was continued and the bleeding stopped. Both were SCI patients with a history of rectal bleeding from haemorrhoids. No other complications developed and the procedure overall was well tolerated.

No procedures were done on patients with absolute contraindications. One procedure was done on a patient with previous bowel surgery $>1$ year with no problems. Four procedures were done on patients with diverticulosis with no complications. As noted above, two procedures were done on patients with rectal bleeding and were tolerated well.

\section{Efficacy}

To evaluate efficacy, again all 398 procedures were evaluated. The procedure was successful in removing stool in all but three patients. Two of these were with the stroke patients who could not tolerate the procedure. One patient was an SCI patient with a very large, distended rectum that could not retain the water despite maximum inflation of the cuff. This was the only failure in an SCI patient.

As already noted, there were a total of 63 procedures done on SCI patients. Of these, 20 patients had only one procedure during their stay, with 19 successful and one unsuccessful. Another 11 patients had multiple procedures. Three of the 11 patients required a second procedure to completely clear the 


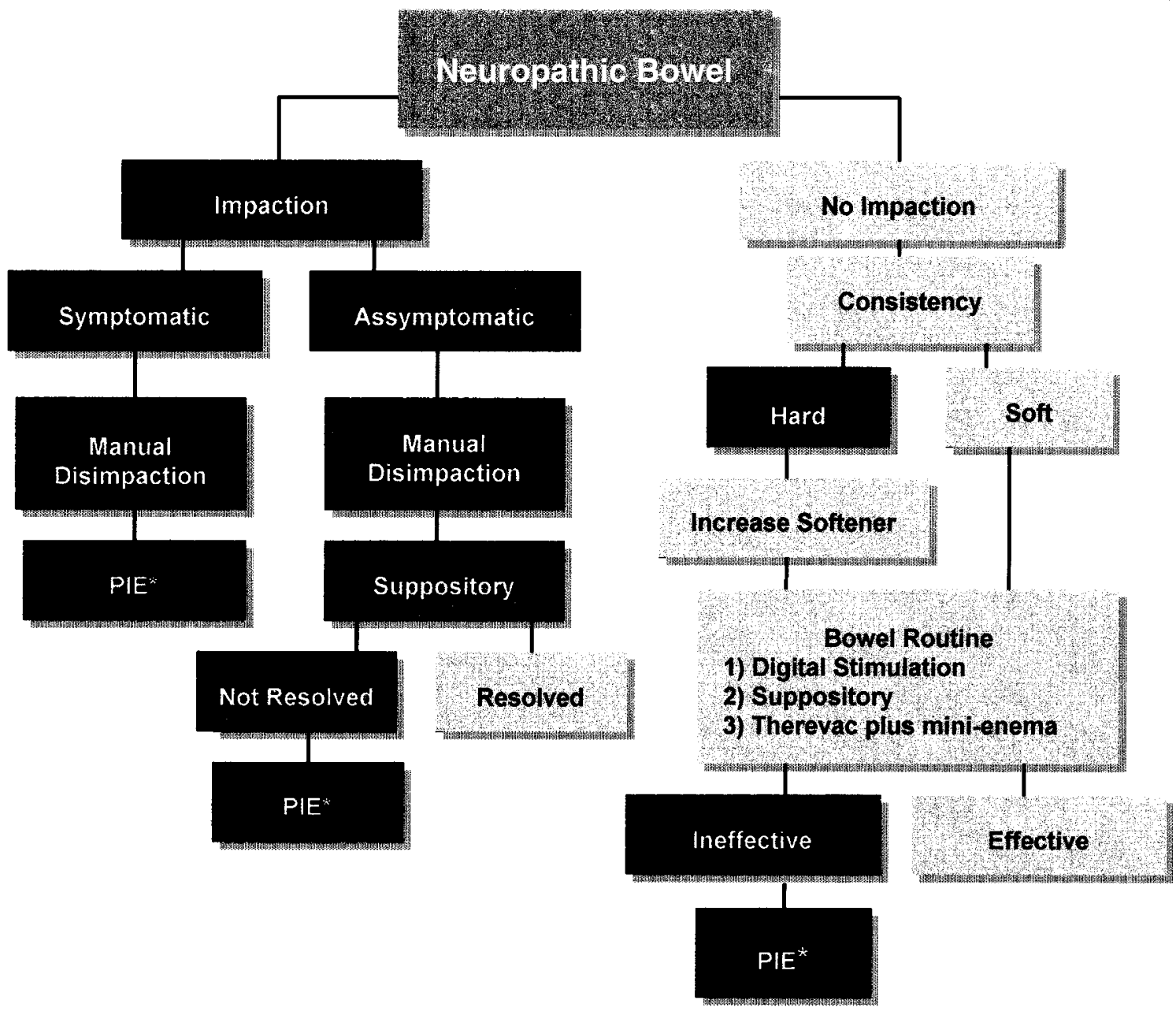

Figure 2

impaction after partial clearing the first procedure. The other 8 patients had more than one procedure on separate occasions for separate impactions or failed bowel routines. Three of these required four procedures, and two went on to regular use of the PIE as outpatients. Table 3 summarizes these results.

\section{Outpatient use}

As previously noted 162 procedures were performed on four outpatients on a regular basis. These patients could not develop an effective bowel routine with the standard digital stimulation, suppositories, or mini enemas. No correctable pathology was found in these patients to explain the failure of the traditional methods. Two of these received their inpatient rehab during the time of the study, and two were referred from the outpatient SCI clinic due to longstanding failed bowel routines.

All procedures were effective and tolerated well. Rectal bleeding occurred with one of these, as noted in Safety.
Case 1 A 22 year old man with an incomplete C5 SCI. He had a two year history of an ineffective bowel routine with frequent involuntary stools, several hospitalizations for impactions, and multiple episodes requiring oral magnesium citrate or go-litely. Each of these required several days of bedrest due to involuntary stools. On regular use of the PIE he has had no hospitalizations or impactions.

Case 2 A 46 year old patient with a C5 incomplete SCI. He averaged three hospitalizations annually for fecal impactions, multiple emergency room visits and frequent involuntary stools. He also has had multiple Autonomic Dysreflexia (AD) reactions related to bowel impactions. He has been on a regular use of the PIE since 12/8/94. He has had no impactions and no hospitalizations since beginning use of the PIE. He has not had any AD reactions during the PIE procedure, and no bowel related AD reactions since beginning regular use, and was able to begin his own business and recently was the recipient of our facility's 'Triumph of the Human Spirit' award for both his 
business achievements and his community service work in peer counselling, and the development of a program where disabled construction trade workers will build ramps for newly disabled individuals. In return, the families of those individuals are asked to help build two other ramps. This has been very successful in allowing those patients and families to see disabled persons in both a functional and social setting.

Case 3 A 71 year old lady with paraparesis due to vascular compromise following abdominal aneurysm surgery. She required several PIE procedures during her SCI rehabilitation, and several months later had a CVA with right-sided hemiplegia. She has required regular use of the PIE since the CVA due to ineffective bowel routine.

Case 4 A 25 year old man with a C5 incomplete SCI. He has had frequent $A D$ reactions caused by minimal bowel distension, and also had frequent involuntary stools. With regular use of the PIE he has had no bowel related $\mathrm{AD}$ reactions and rarely has involuntary stools. He will soon be returning to work in sales. Prevention of unexpected AD reactions and involuntary stools was a key goal required for his return to work. It was also noted that his normal bowel routine prior to the SCI was once weekly bowel movements, however, multiple combinations of medications on a daily, q.o.d., or weekly basis did not prove consistently effective.

\section{Discussion}

The neuropathic bowel is a serious problem for persons with a wide variety of disabilities including stroke, multiple sclerosis, spina bifida and poliomyelitis. It also occurs in some patients with diabetic neuropathy and patients with Alzheimer's disease. ${ }^{5}$ Problems with the neuropathic bowel are probably most significant in those with a spinal cord injury. There are approximately 250000 persons with a severe spinal cord injury in the United States. In one series, $11 \%$ of SCI patients had significant GI complications, and $45 \%$ of those were fecal impactions. ${ }^{6}$

Impactions were seen in $8 \%$ of patients seen within $72 \mathrm{~h}$ of injury. ${ }^{6}$ An extensive review of morbidity among long term survivors of SCI showed an increase in GI complications with age. Annual incidence increased from $5.3 \%$ in those $<30$ years old to $9.0 \%$ in those $40-49$, to $15.3 \%$ in those $>60$ years old. ${ }^{7}$

A variety of methods have been developed for managing impactions that have not responded to the standard bowel routine. Retention enemas two to three times daily have been recommended, ${ }^{8}$ but are frequently ineffective in SCI patients because of inability to retain the enema. Whole gut irrigation has been recommended, but this frequently results in prolonged periods of uncontrolled fecal soiling which is disturbing to patients and disruptive of the rehabilitation program. This prolonged bedrest and soiling may also contribute to decubitus ulcer and urinary tract infections by colonic bacteria. Irrigation with the sigmoidoscope can be effective, ${ }^{9}$ but is a long and costly procedure. Colostomy has even been recommended as an alternative method of handling recurrent impactions. ${ }^{10}$ We have previously reported our experience with a small group of patients. ${ }^{4}$ The PIE procedure was found to be safe and effective. This was also evaluated in a gastrointestinal surgery setting, ${ }^{11}$ and for bowel preparation. ${ }^{12}$ It has also proven safe and effective for use in children. ${ }^{13}$

This is the first study evaluating a new, more portable and less expensive device using the pulsed irrigation evacuation principle. We found that the device was also safe and effective in clearing impactions. Also important is the fact that it was not disruptive to the patient's rehabilitation program, resulting in very few missed therapy sessions. In asymptomatic patients the procedure was performed after completion of the day's therapy. It was tolerated well by all patients with SCI, causing no serious discomfort and no autonomic dysreflexia. Patients expressed a greater degree of confidence in the bowel program, knowing that they had the PIE available should the normal regimen not be effective. A question could be raised concerning the relatively mild nature of some of the impactions treated with the PIE. However, two of the patients treated in our initial study were already being considered for colostomy at the time they were treated with the PIE and had failed all other treatment methods. None of the patients in this study had reached that level of severity. We felt it was preferable to treat these patients early and not risk having to deal with a more significant problem. Severe impactions do cause significant damage to the bowel mucosa. ${ }^{14}$ We hope to show in future studies that early treatment of impactions can lessen long term complications of the neuropathic bowel. In addition we are completing a study of gross and microscopic pathology in long term uses of Pulsed Irrigation Evacuation. Further evaluation of those patients who require long term use is planned including a study of bowel transit time, both with and without the use of the PIE and a study on long term home use.

\section{Conclusions}

The neuropathic bowel is a common and serious problem in persons with spinal cord injuries. Impactions are a significant complication in these persons. They are more frequent in older SCI patients, and are more difficult to treat with the standard bowel routine. Pulsed irrigation evacuation is a safe and effective method of treating these impactions. It should be considered as an alternative in SCI patients who develop impactions, or do not have an effective bowel routine established. It can be effectively used in both an inpatient and outpatient setting. 


\section{References}

1 Madoff R, Williams JG, Caushaj PI. Fecal incontinence. NEJM 1992; 326: $1002-1007$.

2 Weingarten $\mathrm{S}$. The gastrointestinal system and spinal cord injury. Phys Med and Rehab Clinic N.A. 1992; 3: 765-781.

$3 \mathrm{Krogh} \mathrm{K}$ et al. Constipation and incontinence in patients with spinal cord lesions. Surgical Research Unit, University of Aarhus, Denmark. Presented at IMSOP, Atlanta, Georgia, August 18, 1996.

4 Puet T, Phen L, Hurst D. Pulsed irrigation enhanced evacuation: new method for treating fecal impaction. Arch Phys Med Rehabil 1991; 72: $935-936$.

5 Alessi C, Henderson C. Constipation and fecal impaction in the long-term care patient. Clinics in geriatric medicine 1988; 4: 571 588.

6 Gore R, Mintzer R, Calenoff L. Gastrointestinal complications of spinal cord injury. Spine 1981; 6: $538-544$.

7 Whiteneck GG et al. Mortality, morbidity, and psychosocial outcomes of persons spinal cord injured more than 20 years ago. Paraplegia 1992; 30: 617-630.
8 Cefalu C, McKnight G, Pike JI. Treating impaction: a practical approach to an unpleasant problem. Geriatrics 1981; 36: $143-$ 146.

9 Wrenn K. Fecal impaction. NEJM 1989; 321: 658-662.

10 Stone J, Wolfe V, Nino-Murcia M, Perkash I. Colostomy as treatment for complications of spinal cord injury. Arch Phys Med Rehabil 1990; 71: 514-518.

11 Kokoszka J et al. Treatment of fecal impaction with pulsed irrigation enhanced evacuation. Dis Colon Rectum 1994; 37: $161-164$.

12 Chang KJ et al. Per-rectal pulsed irrigation versus per-oral colonic lavage for colonoscopy preparation; a randomized, controlled trial. Gastrointest Endosc 1991; 37: 444-448.

13 Gilges MA, Wagner ML, Barrish JO, McCarroll LR, Healy WM. New treatment for rectal impaction in children: an efficacy, comfort, and safety trial of the pulsed irrigation enhanced evacuation procedure. Journal of Pediatric Gastroenterology and Nutrition 1994; 18: 92 - 95.

14 Gelias P, Schuster MM. Stercoral perforation of the colon: care, report and review of the literature. Gastroenterology 1981; 80: $1054-1058$ 